Maria Effinger*, Leonhard Maylein und Jakub Šimek

\title{
Von der elektronischen Bibliothek zur innovativen Forschungsinfrastruktur
}

\author{
Digitale Angebote für die Geisteswissenschaften an der Universitätsbibliothek Heidelberg
}

https://doi.org/10.1515/bfp-2019-2067

Zusammenfassung: Seit bald 20 Jahren wird an der Universitätsbibliothek Heidelberg kontinuierlich anhand strategisch ausgewählter Projekte eine modulare digitale Infrastruktur aufgebaut, mit besonderem Blick auf die Bedarfe der Geisteswissenschaften. Sie dient der Arbeit mit Digitalisaten, digitalen Medien und Texten und umfasst Werkzeuge für semantische Modellierung, Bildannotation, Textedition sowie wissenschaftliche Publikation. Erschließung mit Normdaten, bibliothekarische Katalogisierung, Verfügbarmachung von Forschungsdaten und Langzeitarchivierung sorgen für Nachhaltigkeit und hohe Attraktivität des Heidelberger Angebots.

Schlüsselwörter: Forschungsinfrastruktur; Digitale Edition; Elektronisches Publizieren; Open Access

\section{From Electronic Library to Innovative Research Infra- structure \\ Digital Services for the Humanities at Heidelberg Univer- sity Library}

\begin{abstract}
For almost 20 years, Heidelberg University Library has been continuously building a modular digital infrastructure based on strategically chosen projects with a special focus on the needs of the arts. It does so by offering digitized reproductions, digital media and texts and by comprising tools for semantic modelling, image annotation, text editions as well as research publications. Indexing using integrated authority data, library cataloguing, exposure of research data as well as long-term archiving ensure sustainability and enhance the attractiveness of the Heidelberg services.
\end{abstract}

Keywords: Research infrastructure; digital edition; electronic publishing; open access

*Kontaktperson: Dr. Maria Effinger, Effinger@ub.uni-heidelberg.de Leonhard Maylein, maylein@ub.uni-heidelberg.de

Dr. Jakub Šimek, simek@ub.uni-heidelberg.de

\section{Die Anfänge: Digitale Faksimiles}

Das in den Jahren 2001 und 2002 durchgeführte DFG-Projekt „Digitalisierung spätmittelalterlicher Handschriften aus der Bibliotheca Palatina“ gab den Startschuss zur digitalen Erschließung des einzigartigen Handschriftenbestands der Universitätsbibliothek Heidelberg, dessen moderne Katalogisierung einige Jahre zuvor (1996) ihren Anfang genommen hatte. Das aus heutiger Sicht bescheidene, damals jedoch pionierartige und wegweisende Vorhaben hatte die Digitalisierung von 27 oberdeutschen bebilderten Handschriften zum Ziel. Durch eine Kooperation mit dem Institut für Europäische Kunstgeschichte erhielt das Projekt mit der ICONCLASS-basierten Inhaltserschließung der Bilder eine ausgeprägte fachwissenschaftliche Komponente über die reine digitale Reproduktion hinaus. ${ }^{1}$ Die erschlossenen Bilder wurden lokal im Rahmen der damaligen „Virtuellen Fachbibliothek Kunstgeschichte“ online veröffentlicht und parallel über Aggregatoren wie die „Handschriftendatenbank“ angeboten. Die Komplettdigitalisate der Handschriften wurden als blätterbare Faksimiles erstmals im Open Access verfügbar gemacht. Während die Digitalisierung selbst bei diesem ersten Projekt noch an der Universitätsbibliothek Graz durchgeführt wurde, konnte für Folgeprojekte schon bald eine eigene Digitalisierungswerkstatt in Heidelberg eingerichtet werden. ${ }^{2}$

In Heidelberg konzentrierte man sich in den 2000erJahren vorerst überwiegend auf die eigenen umfangrei-

1 Bereits bei diesem ersten Projekt wurde angedacht, die damals im Fokus stehenden Handschriften dreier oberdeutscher Skriptorien mit in anderen Bibliotheken aufbewahrten Codices aus denselben mittelalterlichen Werkstätten „virtuell“ zusammenzuführen. Für die Werkstatt Diebold Laubers ist dies erst deutlich später außerhalb Heidelbergs in Form eines stark auf kodikologisch-kunsthistorische Erschließung fokussierten, allerdings nicht alle Digitalisate umfassenden Portals tatsächlich erfolgt: Diebold Lauber - digital (http://wirote.informatik.uni-leipzig.de/mediavistik, zuletzt zugegriffen am 28.02.2019, so auch alle im Folgenden angegebenen Links).

2 Zum ersten Digitalisierungsprojekt und den damaligen Perspektiven vgl. Effinger et al. (2000), Saurma-Jeltsch und Effinger (2001) sowie Effinger et al. (2003). 
chen Sammlungen und den Aufbau einer leistungsfähigen Digitalisierungsinfrastruktur. ${ }^{3}$ Vorrangiges Ziel war die parallel zur katalogisierenden Beschreibung angestrebte Digitalisierung aller 848 in Heidelberg aufbewahrten Codices der Bibliotheca Palatina. Dieses Großprojekt konnte nur mit Unterstützung der Manfred-Lautenschläger-Stiftung in einer realistischen zeitlichen Perspektive angegangen werden. Zur effizienten Bewältigung der Arbeitsprozesse wurde das webbasierte Workflowsteuerungssystem „DWork - Heidelberger Digitalisierungsworkflow“ aufgebaut, ${ }^{4}$ das sich seit 2008 in kontinuierlicher Weiterentwicklung befindet und längst auch von anderen Institutionen genutzt wird. ${ }^{5}$ Die Beweggründe für die Entwicklung von DWork lagen einerseits in der Notwendigkeit, die Qualität der Digitalisate und zugehöriger Metadaten (METS/ MODS) gemäß den damaligen Empfehlungen der DFG sicherzustellen, ${ }^{6}$ andererseits im Wunsch nach einer attraktiven und benutzerfreundlichen Präsentationsform der Handschriften im Einklang mit dem Corporate Design der Universität Heidelberg. Die Kernaufgabe von DWork war und ist die Unterstützung sämtlicher in der Digitalisierung anfallenden Einzelschritte, von der Erstellung der Bildaufnahmen über die Metadatenerfassung und die Einbindung weiterer wissenschaftlicher Erschließungsdaten bis hin zur Generierung der Webpräsentation und der Initiierung der Langzeitarchivierung.

So wie die Anfänge der Heidelberger Digitalisierungsinitiative in enger Verbindung mit einem kunsthistorischen Forschungsprojekt standen, waren auch die folgenden Schritte stark von der Kooperation mit den Universitätsinstituten geprägt. Es war erneut maßgeblich das Kunsthistorische Institut, welches 2005 während seiner Suche nach einer Lösung für die Digitalisierung seiner Diathek zusammen mit der Universitätsbibliothek den Anstoß für den Aufbau einer universitätsweiten Bilddatenbank gab. Weitere Institute und Einrichtungen der Universität beteiligten sich früh am Aufbau der seitdem unter dem Namen heidICON firmierenden Multimediadatenbank, die vom Anfang an auch für die Erschließung der

$3 \mathrm{Zu}$ den technischen Aspekten der frühen Digitalisierungsinfrastruktur in Heidelberg vgl. Maylein und Effinger (2004).

4 Zu den Anfängen von DWork vgl. Effinger et al. (2008) 158-163 sowie Effinger (2010) $81 \mathrm{f}$.

$5 \mathrm{Zu}$ aktuellen Beschreibungen der Software DWork und des gesamten Digitalisierungsworkflows vgl. http://dwork.uni-hd.de sowie https://www.ub.uni-heidelberg.de/helios/digi/tech_workflow.html. 6 In heute aktueller Form vgl. DFG-Praxisregeln „Digitalisierung“ unter http://www.dfg.de/formulare/12_151/index.jsp, für die Fassung von 2009 vgl. http://www.dfg.de/download/pdf/foerderung/pro gramme/lis/praxisregeln_digitalisierung_2009.pdf.
Bebilderung mittelalterlicher Handschriften eingesetzt wurde. ${ }^{7}$

Nachdem in nur drei Jahren Projektlaufzeit 2009 die Digitalisierung und Online-Veröffentlichung aller 848 Heidelberger Palatina-Handschriften erfolgreich abgeschlossen war, beschäftigte sich die Digitalisierungswerkstatt der UB längst nicht mehr nur mit der Onlinestellung von Handschriften. Der Ausbau digitaler Angebote in den von der DFG geförderten Heidelberger Sondersammelgebieten sowie weitere Forschungsprojekte führten u.a. zur Digitalisierung alter Drucke und historischer Zeitschriften. Inhaltliche Schwerpunkte bilden bis heute die Digitalisierung und Erschließung illustrierter Kunst- und Satirezeitschriften des 19. und frühen 20. Jahrhunderts ${ }^{8}$ sowie klassischarchäologischer und ägyptologischer Monografien respektive Zeitschriften aus dem Zeitraum vom 16. bis zum frühen 20. Jahrhundert ${ }^{9}$ und die Bereitstellung digitalisierter Auktionskataloge der Jahre 1901 bis $1945 .{ }^{10}$ Sowohl in der Anfangsphase der Handschriftendigitalisierung als auch in allen Nachfolgeprojekten grenzte sich die UB Heidelberg von der reinen Massendigitalisierung durch eine qualitativ hochwertige Tiefenerschließung ab, die durch die Bereitstellung wissenschaftlich fundierter Metadaten eine intellektuelle Nutzung ermöglicht und den Forschungsdiskurs befördert.

\section{Das Ganze im Blick: Virtuelle Rekonstruktion historischer Bibliotheken}

Bei den bisher genannten Vorhaben waren zunächst die eigenen Buch- und Zeitschriftenbestände Gegenstand der digitalen Erfassung. Anfang der 2010er-Jahre eröffneten sich jedoch neue, darüber hinausweisende Perspektiven, die einerseits in der digitalen Zusammenführung historischer Sammlungen, andererseits in über Heidelberg thematisch hinausgehenden Forschungsprojekten bestanden. Während heute die Zusammenführung verteilter Bildressourcen (via IIIF ${ }^{11}$ ) wenn nicht schon gängige Praxis, dann zumindest - auch dank der immer gängigeren offenen

7 Zur Entstehung und den ersten Funktionen von heidICON vgl. Effinger und Wolf (2005).

8 Vgl. Effinger und Schmidt (2009). Für eine Übersicht der an der UB Heidelberg geförderten Erschließungsprojekte s. https://www.ub. uni-heidelberg.de/wir/projekte.html.

9 https://www.propylaeum.de/themen/rezeption-der-antike. 10 https://www.arthistoricum.net/themen/portale/german-sales. 11 International Image Interoperability Framework, https://iiif.io. 
Lizensierung von Digitalisaten historischer Bestände - ein auf breitem Konsensus basierendes, realistisches Ziel der Bibliothekscommunity ist, war um 2010 besonders in der zwischeninstitutionellen Kommunikations- und Kooperationskultur weitgehend Neuland zu betreten. ${ }^{12}$

Hier war insbesondere die virtuelle Rekonstruktion der vollständigen Handschriftensammlung der ehemaligen Bibliotheca Palatina und deren Bereitstellung im Open Access eine besondere Herausforderung. Der weitaus größte Teil dieser früher weltberühmten Bibliothek befindet sich seit dem Dreißigjährigen Krieg im Vatikan. Bereits für ein verwandtes Projekt, die digitale Zusammenführung der Bibliothek des ehemaligen Klosters Lorsch, konnten die ursprünglich Lorscher, später Heidelberger Palatina-Handschriften dank einer Kooperationsvereinbarung mit der Biblioteca Apostolica Vaticana seit 2010 durch ein Heidelberger Team in Rom digitalisiert werden. ${ }^{13}$ Es folgte (erneut mit der Unterstützung der Manfred-Lautenschläger-Stiftung) die Digitalisierung aller lateinischen Handschriften der Palatina im Vatikan, die 2018 abgeschlossen wurde und von einem Katalogisierungsprojekt begleitet wird. ${ }^{14}$ Aktuell werden - finanziert durch „The Polonsky Foundation“ auch die griechischen Palatina-Handschriften digitalisiert und katalogisiert. Im Fall der Rekonstruktion der Lorscher Bibliothek wurde mit mehr als 70 Institutionen weltweit, auf welche die ehemalige Handschriftensammlung heute verstreut ist, eine Kooperation hinsichtlich der Bereitstellung von Digitalisaten eingegangen.

\section{Mehrwerte schaffen: Digitale Werkzeuge für die Visualisierung und Präsentation von Erschließungsdaten}

Die Anzeige der digitalisierten Seiten ist die primäre Aufgabe der Präsentationsschicht von DWork, aber längst nicht mehr die einzige. Inzwischen sind eine Reihe weiterer Funktionen hinzugekommen, die der Kontextualisie-

12 Als eines der ersten - Nicht-Heidelberger - Pionierprojekte zur erfolgreichen digitalen Zusammenführung einer (in diesem Fall einzigen) physisch dislozierten Handschrift kann das Projekt „Codex Sinaiticus“ (http://www.codexsinaiticus.org) genannt werden. Das Projekt vereint seit 2009 die auf vier Bibliotheken verteilte Handschrift.

$13 \mathrm{Zu}$ diesem Projekt „Bibliotheca Laureshamensis - digital“ vgl. Effinger (2011) sowie Effinger et al. (2012).

14 Vgl. Effinger und Zimmermann (2015). rung der angezeigten Digitalisate dienen, Erschließungsergebnisse sichtbar machen und weitere Forschung zielgerichtet befördern.

So können in die DWork-Präsentation zu jeder Seite weitere Informationen aus beliebigen Quellen importiert werden, die im Text- oder HTML-Format auf jeweils dedizierten Reitern neben dem Faksimile präsentiert werden. Die UB Heidelberg nutzt diese Funktion beispielsweise, um zugehörige Kataloginformationen direkt neben den digitalen Faksimiles der Heidelberger Papyrus-Sammlung ${ }^{15}$ anzubieten. Im großen Maßstab wird dies zudem eingesetzt, um bei der Darstellung der jeweiligen Seite automatisch Erschließungsinformationen zu Miniaturen oder Wasserzeichen aus der universitären Objekt- und Multimediadatenbank heidICON zu ergänzen, wo diese detailliert erschlossen werden.

Die Wasserzeichen werden mit einem eigens zu diesem Zweck erworbenen Wasserzeichenscanner mit Infrarot-Technologie (ATWISE 5242) ${ }^{16}$ aufgenommen. Dank dieser Technik ist es möglich, Schrift aus Eisengallustinte, dem im Mittelalter am meisten verbreiteten Schreibstoff, nahezu transparent erscheinen zu lassen und in den Aufnahmen „auszublenden“. Hierdurch entstehen unter für die Handschriften schonenden Bedingungen ausgesprochen deutliche und unbeeinträchtigte Abbilder der Marken. ${ }^{17}$ Die Erschließung der Wasserzeichen (also deren Identifizierung und Beschreibung anhand einschlägiger Repertorien und Datenbanken) erfolgt im Anschluss. ${ }^{18}$ In einem abschließenden Schritt wird der Datensatz in heidICON mit einem externen Link zu dem identifizierten Wasserzeichen in der entsprechenden Wasserzeichen-Datenbank $^{19}$ versehen, so dass der Benutzer jederzeit eine anhand des Wasserzeichenbefundes erfolgte Datierung

15 https://www.ub.uni-heidelberg.de/helios/digi/hd_papyrus.html. 16 Entwicklung der Universitätsbibliothek Graz. ATWISE steht für Austrian Watermark Imaging System, 5242 weist darauf hin, dass die Blattgröße maximal $52 \times 42 \mathrm{~cm}$ betragen darf, s. http://eprints.rclis.or $\mathrm{g} / 22999$.

17 Die Aufnahmen der Wasserzeichen erfolgen immer von der Versoseite aus. Es werden jeweils alle Wasserzeichen einer Handschrift aufgenommen und in heidICON eingespielt, auch wenn sich identische Motive wiederholen. Um eine möglichst hohe Identifizierbarkeit mit den gebräuchlichen Wasserzeichenrepertorien und -datenbanken zu gewährleisten, werden lediglich die Wasserzeichen von Handschriften im Folio- und Quartformat digitalisiert, da bei kleineren Formaten die Zerstückelung der Wasserzeichen eine eindeutige Vergleichbarkeit unmöglich macht.

18 In einem ersten Schritt werden die Wasserzeichen-Motive anhand einschlägiger Repertorien und Nachweissysteme bestimmt und idealerweise nachfolgend mit dort bereits nachgewiesenen datierten Referenzen identifiziert (identisch, ähnlich, vergleichbar).

19 Bspw. http://www.wasserzeichen-online.de/wzis/index.php. 
der Handschrift oder eines Handschriftenfaszikels nachvollziehen kann. Die Erschließungsdaten zu den Miniaturen und Wasserzeichen werden täglich via OAI-PMHSchnittstelle aus heidICON abgerufen und in die DWorkPräsentation eingespielt. Sie stehen damit in der Präsentation auch für die Volltextsuche zur Verfügung. Bei den Wasserzeichen wird zusätzlich der Wasserzeichenscan selbst angezeigt.

Neben dieser Anreicherung der Faksimile-Anzeige ist es möglich, in Form von Annotationen zum Digitalisat Verknüpfungen $\mathrm{zu}$ in heidICON abgelegten Spezialaufnahmen von beispielsweise beschädigten oder schwer lesbaren Seiten einzubinden. Dazu gehören momentan einige UV-Aufnahmen mittelalterlicher Handschriftenfragmente, ${ }^{20}$ bald aber auch Infrarot-, Durchlicht-, Streiflicht- sowie Röntgen-Fotografien, die in Kooperation mit anderen Bibliotheken im Auftrag von Editionsprojekten entstanden sind. Demnächst werden darüber hinaus Mikroskopaufnahmen sowie multispektrale und hyperspektrale Bilder der Anzeige einiger Digitalisate an die Seite gestellt werden. $\mathrm{Zu}$ einigen, anhand wissenschaftlicher Fragestellungen ausgewählten Handschriften werden Verknüpfungen $\mathrm{zu}$ in heiDATA, dem Forschungsdaten-Repositorium der Universität Heidelberg (s.u.), publizierten Ergebnissen von Tintenanalysen eingepflegt.

Das hierfür eingesetzte Annotations- und Kommentarmodul wurde an der UB Heidelberg basierend auf der W3C-Empfehlung „Web Annotation Data Model“ (ehemals Open Annotation Collaboration) als System zur Annotation verschiedener webbasierter Publikationsdienste entwickelt. Es ist nahtlos in die DWork-Präsentation integriert, wird aber auch in den E-Book- und E-Journal-Plattformen und demnächst auch in heidICON und den WissKI-Anwendungen eingesetzt (s. u.). Über einen Formeneditor, der die grafische Markierung und Auszeichnung von Rechtecken, Kreisen und Polygonen in den Digitalisaten ermöglicht, können Textstellen oder Abbildungen lokalisiert, annotiert und kommentiert werden. Darüber hinaus können die Inhalte der digitalen Faksimiles mit weiteren Zusatzinformationen, zum Beispiel Georeferenzierungen oder Plänen, verbunden werden. ${ }^{21}$

So können - neben den systematisch und auch normdatenbasiert erfassten Informationen beispielsweise $\mathrm{zu}$

20 Vgl. z.B. https://digi.ub.uni-heidelberg.de/diglit/hab_mss404-9novi-6/0002.

21 Über die Annotationen können so semantische Verknüpfungen auf externe Objekte erstellt werden. Durch die Entwicklung einer IIIF-Metadaten-API wird das IIIF-Protokoll (International Image Interoperability Framework) unterstützt, sodass auch Bildausschnitte eindeutig referenziert werden können.
Schriftbild, Materialität oder Lokalisierung - auch am digitalen Faksimile gewonnene Erkenntnisse sofort innerhalb der Forschungscommunity in Form von Mikropublikationen zur Diskussion gestellt werden. Die Annotationen selbst können von anderen Nutzern kommentiert werden. Der wissenschaftliche Diskurs kann somit direkt an der historischen Quelle stattfinden. Um eine Zitierfähigkeit der Annotationen $\mathrm{zu}$ erreichen, sind diese mit dem Namen ihres Urhebers versehen sowie einer automatischen Versionierung unterworfen. Bei Bedarf können sie mit DOIs (sowohl für die Annotation als Ganzes als auch für die einzelnen Versionen) versehen werden. Zur Qualitätssicherung wurde das Annotationssystem so konfiguriert, dass das Annotieren oder Kommentieren nur nach vorheriger Authentifizierung (via Shibboleth / eduGAIN) und einer Freischaltung seitens eines Redaktionsteams möglich ist.

Eingesetzt wird dieses Werkzeug aktuell auch im Bereich der Bildanalyse mittelalterlicher Handschriften ${ }^{22}$ sowie für die Verknüpfung von Textstellen mit Transkriptionen oder Übersetzungen. ${ }^{23}$ Auch Kommentare zu gedruckter Forschungsliteratur sind möglich. ${ }^{24}$ Perspektivisch soll das Annotationsmodul auch für die Identifizierung von handschriftlichen Randnotizen in historischen Auktionskatalogen und sogar für Rezensionen elektronischer Publikationen eingesetzt werden. Durch die Katalogisierung der Annotationen im SWB/K10plus erlangen diese den Status zitier- und präsentierbarer Mikropublikationen, wodurch sie für wissenschaftliche Curricula relevant werden.

Die Bereitstellung von Digitalisaten bietet Wissenschaftlern weltweit die Möglichkeit, bequem vom eigenen Arbeitsplatz Handschriften studieren zu können, ohne zum Original reisen zu müssen. Mit der zusätzlichen Visualisierung von Lagenstrukturen - wie auch mit der zuvor erwähnten Präsentation und Erschließung von Wasserzeichen und der Einbindung von Spezialaufnahmen - soll die Remote-Nutzung noch weiter vorangetrieben werden, auch um damit noch mehr als bisher die Originale schonen zu können (Abb. 1). Die Visualisierung geht über die (meist nicht eindeutige) herkömmliche Lagenformel deutlich hi-

22 So bisher v.a. im Projekt „Welscher Gast digital“, vgl. z. B. https:// digi.ub.uni-heidelberg.de/diglit/fbg_membI120/0018.

23 Siehe z.B. beim „Lorscher Codex“, https://archivum-lauresha mense-digital.de/view/saw_mainz72.

24 So zum Beispiel ein ausführlicher Kommentar zu Joseph Braun (Der christliche Altar in seiner geschichtlichen Entwicklung, Band 1, 1924) von Sebastian Bock (Zur Altarkammer des Hochaltars im Münster U.L.F. in Freiburg i. Br.) https://doi.org/10.11588/anno.diglit.AKh B1XIyTCGjxPvtw7JEIA. 


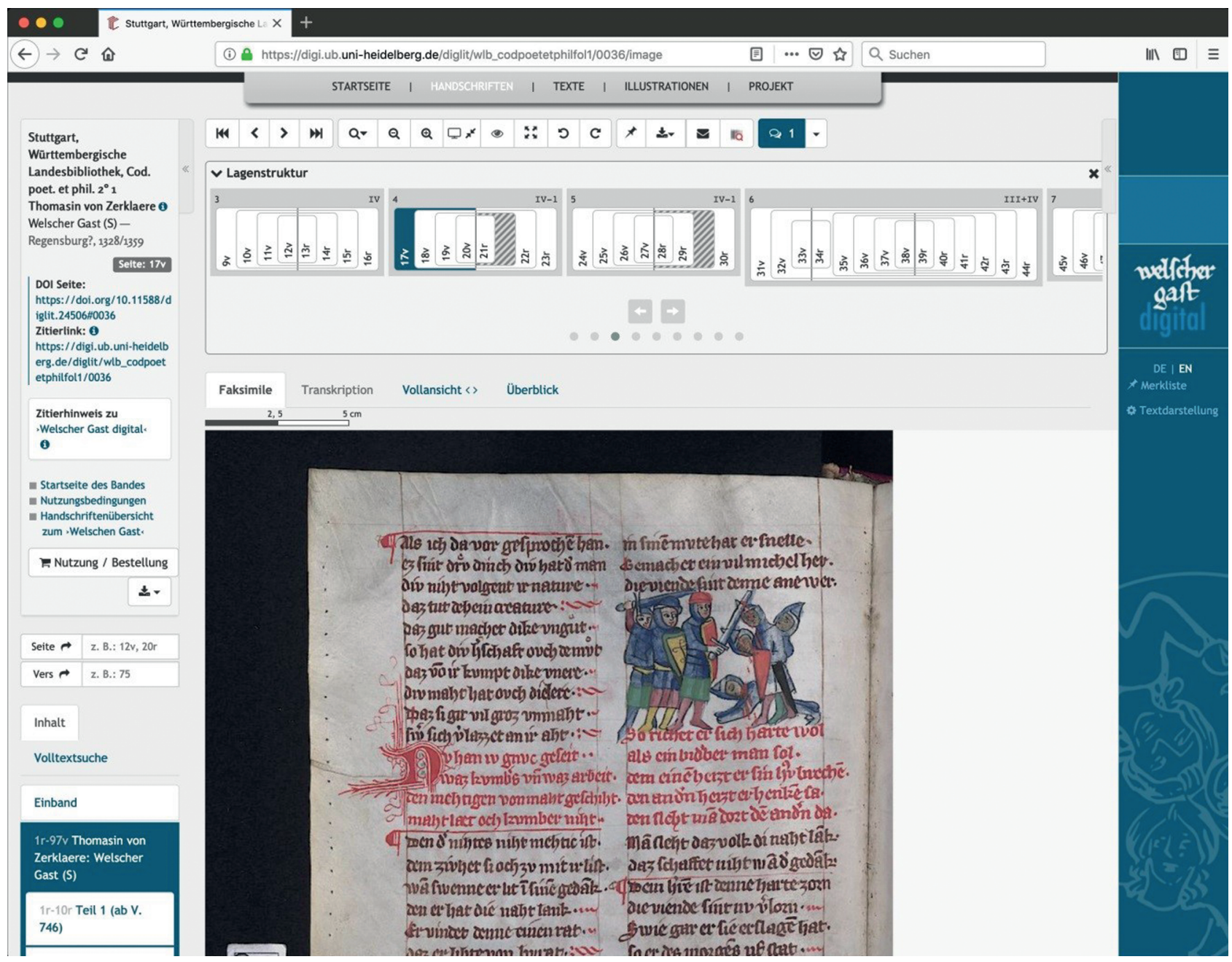

Abb. 1: Visualisierung der Lagenstruktur am Beispiel einer Handschrift der Württembergischen Landesbibliothek Stuttgart (Editionsprojekt „Welscher Gast digital“)

naus. Die Lagenstruktur insgesamt wird mitsamt fehlenden oder eingefügten (Doppel-)Blättern bzw. ganzen Lagen in einer TEI-konformen ${ }^{25}$ XML-Struktur vollständig erfasst und anhand eines neuartigen visuellen Modells dargestellt. Für die einzelnen Blätter können zudem weitere Informationen, wie z.B. das Vorhandensein von Wasserzeichen, optisch signalisiert werden. Für die Erzeugung der komplexen zugrunde liegenden XML-Gefüge wurde ein benutzerfreundlicher grafischer Lagenstruktureditor entwickelt und in das Workflowprogramm DWork integriert. Mit seiner Hilfe werden durch Eingabe und Transformation herkömmlicher Lagenformeln und - wenn nötig - manuelle Anpassung durch Drag-and-Drop (bei Einfügungen und Lücken) XML-Dateien generiert, die anschließend als Grundlage für die Visualisierung dienen.

25 Zur Text Encoding Initiative (TEI) vgl. http://www.tei-c.org.

\section{Die Texte im Fokus: Digitale Editionen}

Auf die Erfahrungen, welche die UB Heidelberg im „Lorscher" Projekt bei der Erstellung digitaler Sammlungen mit Aufnahmen physisch verteilter Bestände gesammelt hat, kann sie auch bei den digitalen Editionsprojekten aufbauen, in die sie seit 2014 in unterschiedlichen Kooperationsformen engagiert ist. Seit 2018 werden diese Aktivitäten unter dem Namen heiEDITIONS vereinigt. ${ }^{26}$ Dabei geht es in der Regel um die digitale Zusammenführung von Handschriften (und anderen schrifttragenden Artefakten) als Textzeugen eines zu edierenden Werkes. Eine Pilotfunktion beim Aufbau entsprechender Kompetenzen

26 https://www.ub.uni-heidelberg.de/publikationsdienste/digita le_editionen.html 


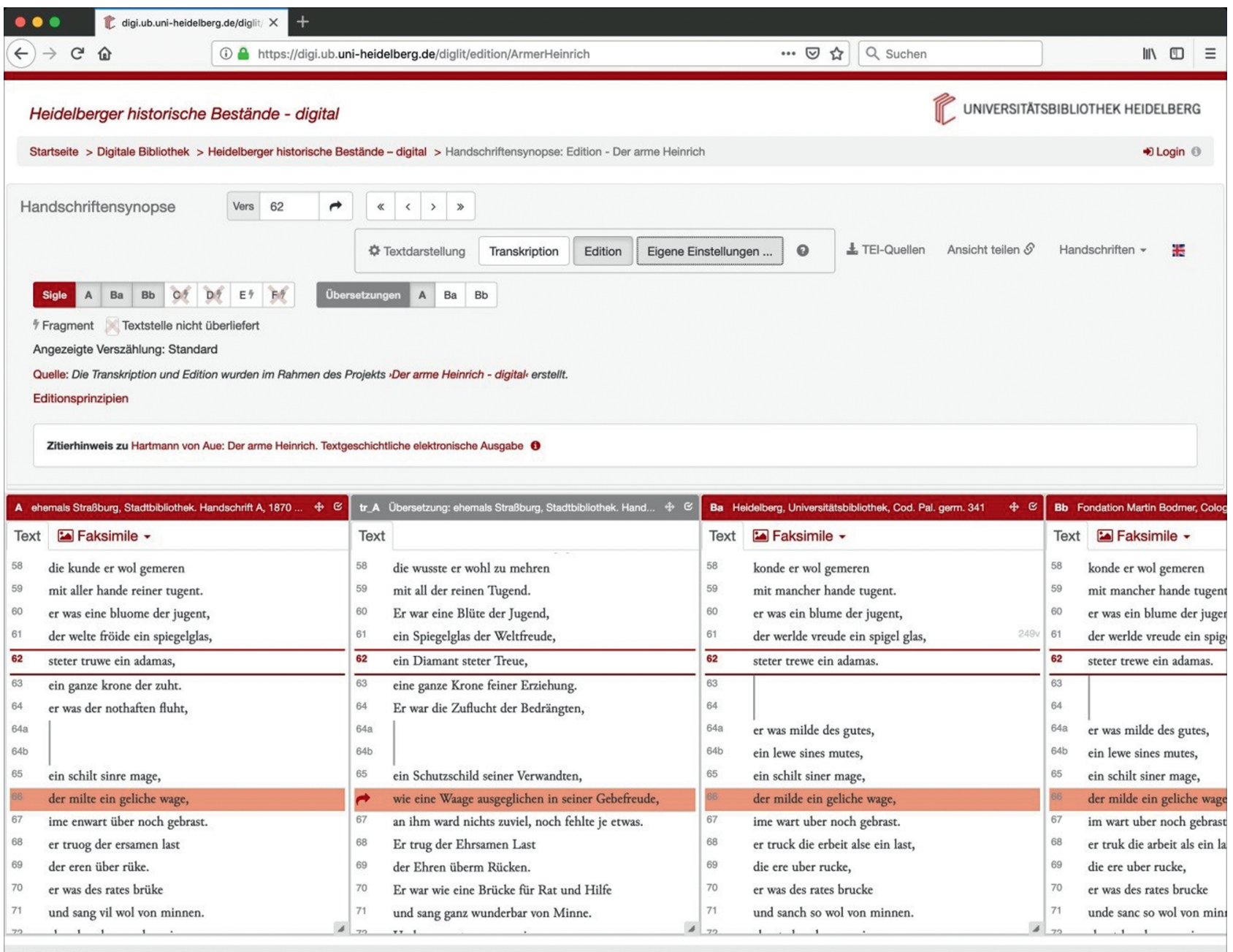

Abb. 2: Synoptische Darstellung mehrerer Textzeugen und einer Übersetzung mit konfigurierbarer Textanzeige (Editionsprojekt „Der arme Heinrich - digital“)

und infrastruktureller Komponenten kam dabei dem Projekt „Welscher Gast digital“ zu, einer Kooperation mit dem Sonderforschungsbereich 933 „Materiale Textkulturen" in Heidelberg. ${ }^{27}$ Bei diesem Projekt wurden an der UB erstmals TEI-kodierte Transkriptionstexte als Pendant zum digitalen Faksimile in den Handschriftenviewer eingebunden. Neben diesem kamen in den nachfolgenden Jahren weitere Editionsprojekte hinzu, für welche die TEIInfrastruktur kontinuierlich ausgebaut wurde, um nicht nur unterschiedliche Editionsgegenstände (Papyri, Gelehrtenkorrespondenzen, religiöse und rechtsgeschichtliche Quellen aus Nepal, demnächst auch hebräische Mikrografien), sondern auch verschiedene editorische Paradigmen abbilden und bedienen zu können, so zum

27 https://digi.ub.uni-heidelberg.de/wgd.
Beispiel für die Edition des „Armen Heinrich“ ${ }^{\text {28 }}$ und der „Kaiserchronik“. ${ }^{29}$

Das dabei eingesetzte Editionsmodul stellt das umfangreichste Add-on der DWork-Präsentation dar. Es integriert sich einerseits nahtlos in die Präsentation der Digitalisate, kann andererseits jedoch auch eigenständig betrieben werden. Mithilfe dieses Moduls können in Verbindung mit den Digitalisaten Transkriptionen und unterschiedlich stark edierte Texte angezeigt werden, die direkt neben dem digitalen Faksimile als Pop-up oder in einem eigenen Reiter untergebracht werden können. Liegen die Transkriptionen bzw. Editionen verschiedener Textzeugen eines Werkes vor, können diese in einer Synopse einander gegenübergestellt werden. In beiden Fällen erlaubt eine Vielzahl von Anzeigeoptionen sowohl dem Herausgeber

28 https://digi.ub.uni-heidelberg.de/ahd. $29 \mathrm{https} / /$ digi.ub.uni-heidelberg.de/kcd. 
als auch dem Leser eine hohe Konfigurierbarkeit der Textdarstellung (Abb. 2).

Besonderer Wert wurde auf die Verarbeitung editorischer Eingriffe, Normalisierungen und Anmerkungen gelegt. Herausgeber können hier nicht nur bestimmen, wie der zugehörige Apparat aufgebaut und dargestellt wird, sondern auch, welche Anzeigemöglichkeiten dem Leser an die Hand gegeben werden. Neben dem klassischen Fußnotenapparat können dabei Varianten gewählt werden, welche die Möglichkeiten digitaler Editionen umfänglich ausnutzen und die zugehörige Information direkt am „Ort des Geschehens“ - z. B. als Pop-up oder aufklappbar über der jeweiligen Zeile - anzeigen. Der Leser kann wiederum entscheiden, welche textuellen Elemente dem historischen Artefakt und welche der editorischen „Schicht“ entnommen und in die Anzeige integriert werden sollen, etwa im Bereich der Interpunktion. Neben einer Reihe einzeln steuerbarer Optionen hat er aber auch grundsätzlich die Wahl, sich ausschließlich den Originaltext oder ausschließlich den Editionstext ohne störende Kennzeichnung der Eingriffe oder Normalisierungen ausgeben zu lassen. Unterstützt wird außerdem auch die Einbindung von Übersetzungen der edierten Texte in die Präsentation.

Vom Editionsmodul können prinzipiell beliebige Varianten TEI-P5-codierter Transkriptionen verarbeitet werden. Jeder Herausgeber ist frei in der Entscheidung, wie tief dabei die Erschließung erfolgen soll. Auch bereits im Rahmen anderer Projekte erfasste TEI-Daten können nachträglich übernommen werden. ${ }^{30} \mathrm{Da}$ die TEI sehr viele Freiheiten lässt, gibt es für praktisch alle zu erfassenden Texteigenschaften eine Vielzahl an Codierungsmöglichkeiten. Für die Verarbeitung im DWork-Editionsmodul müssen diese Codierungen vereinheitlicht werden. Hierzu durchlaufen alle Transkriptionstexte vor dem Einspielen eine Pipeline verschiedener Transformationsschritte, welche die TEI-Daten schließlich in ein intern unterstütztes TEIFormat überführen. Diese Daten werden (zusammen mit den ursprünglichen, also von den Herausgebern bereitgestellten TEI-Dateien) in einer XML-Datenbank (eXist$\mathrm{db}^{31}$ ) gespeichert. Die Konvertierung in HTML (in Echtzeit bei einer Benutzeranfrage) übernimmt eine XQuery-Applikation unter eXist-db. Die so entstandenen HTML-Daten enthalten dabei alle Informationen, die für die verschiedenen Anzeigeoptionen benötigt werden. In Abhängigkeit der gewählten Optionen übernimmt eine JavaScript-An-

30 So z. B. für die Heidelberger Papyrussammlung (https://www.ub. uni-heidelberg.de/helios/digi/hd_papyrus.html), für die die Transkriptionen in TEI bereits in der internationalen Datenbank papyri. info erfasst wurden.

31 http://exist-db.org. wendung in Kombination mit editionsspezifisch anpassbaren CSS-Stylesheets die Aufbereitung der Texte. Neben der für die dynamische Online-Präsentation benötigten Speicherung in der eXist-db-Datenbank werden bei abgeschlossenen Projekten die basalen TEI-Dateien zusammen mit grundlegenden Metadaten im Forschungsdatenrepositorium heiDATA (s.u.) abgelegt, mit permanenten Identifiern veröffentlicht und für die Langzeitarchivierung bereitgestellt. Solche dauerhaften Ablagen sind freilich versionierbar, wodurch selbst bei abgeschlossenen Projekten Revisionen und „Neuauflagen“ möglich sind.

Das Editionsmodul wird derzeit mit hohem Tempo weiterentwickelt. Die jüngste Erweiterung besteht in der Unterstützung von ebenfalls TEI-basierten Registern für Personen, Körperschaften und Orte, aber auch von Glossaren und Literaturverzeichnissen. Die Registerinformationen können direkt in der Transkription über Pop-ups eingeblendet werden und dynamisch mit Daten aus der Gemeinsamen Normdatei (GND) und Bildern aus Wikimedia Commons oder heidICON angereichert werden. Die Recherche in den Registern ermöglicht einen weiteren, gezielten Einstieg in die Edition, andererseits können Registerauszüge aber auch von der Edition aus aufgerufen werden. ${ }^{32}$ In den Texten ausgezeichnete Georeferenzen können in Kürze auch auf aktuellen oder historischen Karten visualisiert werden.

\section{Wissen, was gemeint ist: Nomenklatorische Standardbildung für digitale Editionen}

Während sich TEI zunehmend als technischer Textkodierungsstandard für digitale Editionen etabliert, besteht gleichzeitig eine große Diversität bei der Angabe kodikologischer, paläografischer und editorischer Befunde im TEICode. Das führt in der Praxis häufig zu Ad-hoc-Lösungen einzelner Editionsprojekte oder zu präzisierenden Vorgaben innerhalb institutioneller Infrastrukturen, die auch eine mehr oder minder standardisierende Ausstrahlung auf andere Anwender haben können. So wirkt in Deutschland etwa das Deutsche Textarchiv mit seinem DTA-

32 Erprobt wird diese neue Funktionalität aktuell in dem von der Thyssen-Stiftung finanzierten Projekt zur Erstellung einer digitalen Edition des Briefwechsels der Familien Mommsen und WilamowitzMoellendorff, vgl. https://www.propylaeum.de/themen/mommsenwilamowitz-moellendorff. 


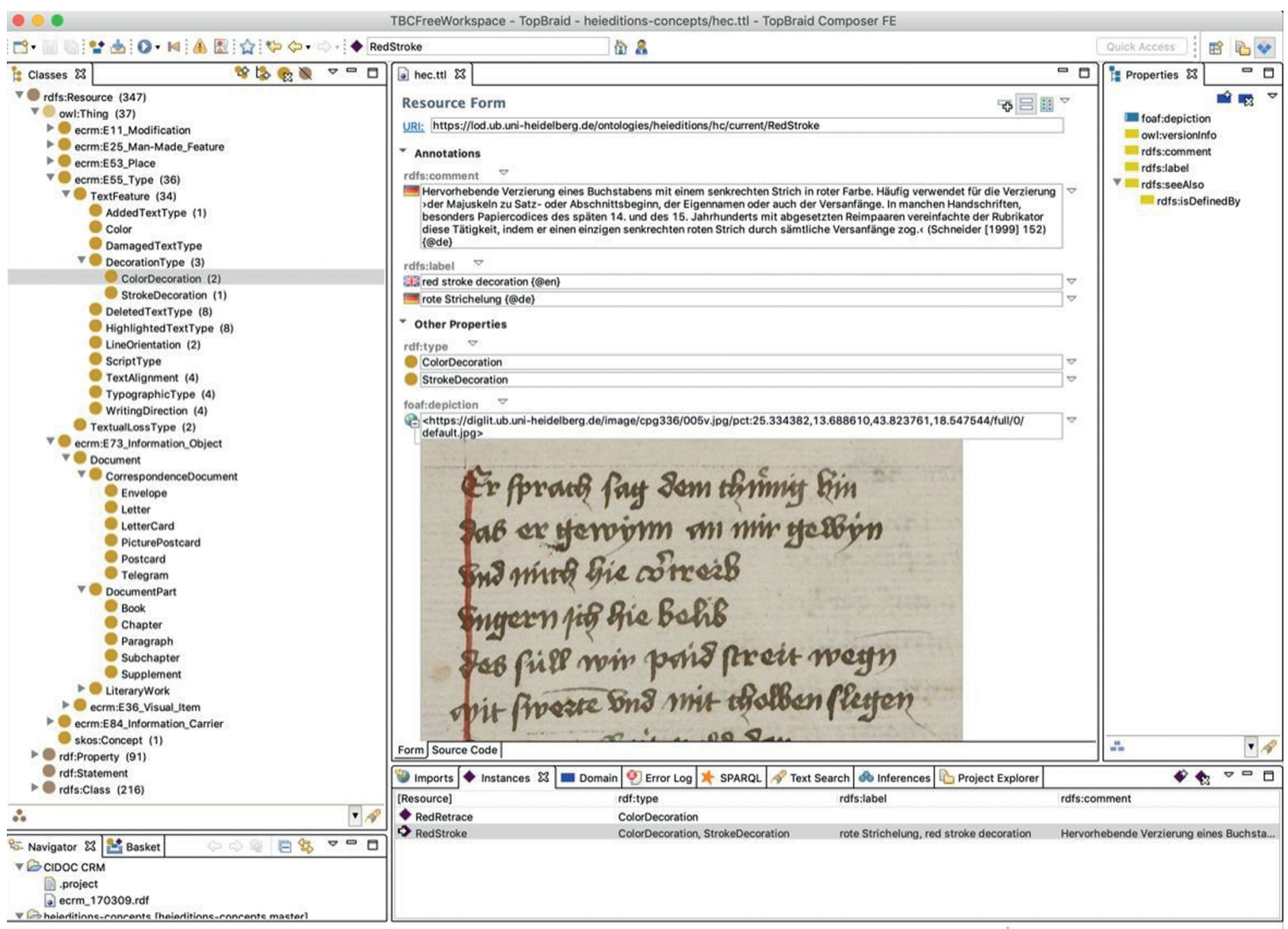

Abb. 3: Der Ontologieeditor „TopBraid Composer“ während der Arbeit an der Ontologie heiEDITIONS Concepts

Basisformat - allerdings bisher vor allem nur für neuzeitliche Drucke - modellbildend. Im Projekt „Welscher Gast digital“ wurden seit 2014 mit einem „TEI-Handbuch“ klare Kodierungskonventionen festgelegt. Diese Konventionen, bislang nur bezogen auf Handschriften des 13. bis 15. Jahrhunderts, dienen mittlerweile auch als Vorbild für eine Reihe weiterer internationaler Editionsprojekte.

Basierend auf diesen Erfahrungen und mit einem viel breiteren Spektrum von Anwenderprojekten im Blick wird an der UB aktuell die editorische Ontologie heiEDITIONS Concepts ${ }^{33}$ entwickelt, die klar definierte Typologien und Nomenklaturen für die Bezeichnung von editorisch relevanten Phänomenen nicht nur in interdisziplinärer $\mathrm{Zu}$ sammenarbeit mit verschiedenen Kooperationspartnern sammelt und dokumentiert, sondern in der für jedes Konzept auch ein eindeutig über ihren URI adressierbarer Datensatz angelegt wird. Diese Datensätze werden technisch

33 https://www.ub.uni-heidelberg.de/publikationsdienste/heiedi tions-concepts.html. im Ontologie-Format OWL strukturiert, untereinander verknüpft und - wo sinnvoll - auch mit der GND verlinkt (Abb. 3). In TEI-Dateien wird es dann möglich, anstatt willkürlich festgelegter Attributwerte auf extern definierte URIs zu verweisen und auf diese Weise nicht nur die methodische Sicherheit bei Editoren, sondern auch die Interoperabilität der Editionsdaten zu erhöhen. Das Zusammenspiel dieser Angaben mit den von der TEI vorgegebenen Strukturen der Elemente und Attribute wird durch ein eigenes XML-Schema (heiEDITIONS Schema) geregelt. ${ }^{34}$

34 Das erste Editionsprojekt, bei dem in der TEI-Kodierung vom heiEDITIONS-Schema und Verweisen auf die in heiEDITIONS Concepts bereitgestellten Definitionen Gebrauch gemacht werden soll, ist „Welscher Gast digital“. Die dort bereits erfassten Daten werden aktuell in das neue Format migriert. 


\section{Elektronisches Publizieren: Dokumentenserver, E-Books und E-Journals im Open Access}

Die Aktivitäten im Bereich digitaler Editionen waren an der UB Heidelberg nicht einfach „Folge“-Schritte, die nach der reinen Quellen-Digitalisierung nahelagen, sie stehen vielmehr im Kontext von bereits seit Längerem im Hause vorangetriebenen digitalen Publikationsaktivitäten. Bereits seit dem Jahr 2000 betreibt die UB mit heiDOK einen universitären Dokumentenserver, der als Erstveröffentlichungsplattform vor allem für Dissertationen begann und sich längst als generisches Repositorium für „grüne“ oder „goldene“ Open-Access-Veröffentlichungen etabliert hat. ${ }^{35}$ Als eine der Triebfedern für den Ausbau der Publikationsdienste erwiesen sich die Sammelschwerpunkte in Heidelberg, die Virtuellen Fachbibliotheken „arthistoricum.net“ für die Kunstgeschichte, „Propylaeum“ für die Altertumswissenschaften sowie „Safiva“ für Südasien. Die bereits 2007 eingerichteten Dokumentenserver ART-Dok, ${ }^{36}$ PropylaeumDOK ${ }^{37}$ und Safiva-DOK ${ }^{38}$ und bedienen neben Erstpublikationen u.a. Schriftenreihen mit gebündelten Zweitveröffentlichungen namhafter Wissenschaftler. ${ }^{39}$ Ebenfalls im Kontext der inzwischen von der DFG zu „Fachinformationsdiensten" (FID) umfirmierten Portale wurde fachspezifische Plattformen für Open-Access-Zeitschriften aufgebaut. ${ }^{40} \mathrm{Im}$ Fokus steht hier zum einen die Unterstützung der Herausgeber bei der Transformation gedruckter Zeitschriften in ein Open-Access-Publikationsmodell, zum anderen aber auch die Möglichkeit der Neugründung genuiner E-Journals im goldenen Open Access. Insgesamt hostet die UB mit der Open-Source-Software „Open Journal System“(OJS) des internationalen „Public Knowledge Project“ (PKP), die von Heidelberg aus aktiv mitentwickelt wird, mittlerweile weit über 100 E-Journals. ${ }^{41}$

$35 \mathrm{Zu}$ den Anfängen von heiDOK vgl. Effinger und Bonte (2001). https://archiv.ub.uni-heidelberg.de.

36 http://archiv.ub.uni-heidelberg.de/artdok.

37 http://archiv.ub.uni-heidelberg.de/propylaeumdok.

38 Heute unter dem Titel „CrossAsia-Repository“ http://crossasia-re pository.ub.uni-heidelberg.de.

39 Vgl. Effinger (2013) 52f.

40 https://www.arthistoricum.net/publizieren/e-journals-mit-ojs, https://www.propylaeum.de/publizieren/propylaeum-ejournals/, https://crossasia.org/service/crossasia-e-publishing/crossasia-ejour nals.

41 https://www.ub.uni-heidelberg.de/service/openaccess/journals. html, vgl. Effinger und Büttner (2015). Zu den vielfältigen kunsthistorischen Publikationsmöglichkeiten unter dem Dach des FIDs arthistoricum.net vgl. auch Effinger (2018).
Einen Meilenstein in den Publikationsaktivitäten des Hauses bildete 2015 die im Auftrag der Universitätsleitung erfolgte Gründung des Verlags Heidelberg University Publishing (heiUP) und einer entsprechenden Abteilung „Publikationsdienste“ an der UB. Der primär digital ausgerichtete Verlag bietet mit einer Print-on-Demand-Option ein hybrides Publikationsmodell und setzte von Anfang an auf die Sicherung einer hohen wissenschaftlichen wie formalen Qualität. Ein wissenschaftlicher Beirat, Double-BlindPeer-Review, professionelles Verlagslektorat und -satz garantieren die Hochwertigkeit der „goldenen“ Open-Access-Titel. Etwas niedrigschwelliger, da ohne Peer-Review und im Regelfall mit Autorensatz auskommend, ist die Tochterplattform heiBOOKS; die drei FIDs verfügen ebenfalls über entsprechende eigene E-Book-Plattformen inkl. entsprechender Print-on-Demand-Angebote. ${ }^{42}$ Für die Produktion von Monografien und Sammelbänden kommt das mit OJS eng verwandte System Open Monograph Press (OMP) zum Einsatz.

Zunehmend ausgebaut wird die selbstentwickelte Workflow-Steuerungssoftware „Heidelberg Monograph Publishing Tool“ (heiMPT). Dieses System ermöglicht eine zeitgemäße crossmediale Buchherstellung (mit Print-, HTML-, PDF- und ggf. EPUB als Outputformaten) auf medienneutraler XML-Basis. ${ }^{43}$ Ein Schwerpunkt der aktuellen Entwicklung liegt dabei auf sog. Enhanced Publications, bei denen die zugrunde liegenden Forschungsdaten und ggf. multimediale Inhalte mit dem wissenschaftlichen $\mathrm{Pu}$ blikationstext technisch robust und zugleich benutzerfreundlich verknüpft werden. Durch die Integration der Forschungsdaten in die wissenschaftliche Publikation soll einerseits die Nachvollziehbarkeit und Überprüfbarkeit der präsentierten Ergebnisse erhöht, andererseits aber auch die Sichtbarkeit der primären Daten selbst gefördert werden.

\section{Semantische Netzwerke: Dynamisches Publizieren von Text und Bild}

Im Zentrum der aktuellen Aktivitäten der UB Heidelberg im Bereich des elektronischen Publizierens steht die Weiterentwicklung der dynamischen und kollaborativen $\mathrm{Pu}$ -

42 arthistoricum.net - ART-Books (https://books.ub.uni-heidelberg. de/arthistoricum), Propylaeum-eBooks (http://books.ub.uni-heidel berg.de/propylaeum), CrossAsia-eBooks (https://crossasia-books.ub. uni-heidelberg.de/xasia).

43 Vgl. Effinger et al. (2018). 
blikationsmöglichkeiten. Strukturierte wissenschaftliche Texte sollen mit Bildern, Karten oder 3D-Visualisierungen verknüpft werden können. Durch eine ontologiebasierte Datenhaltung in einem Triple Store stehen Forschungsergebnisse mittels Linked Data weltweit zur Verknüpfung mit anderen Datenrepositorien bereit. Prototypisch erprobt wird dieses Format derzeit u. a. mit der Bereitstellung einer Publikationsplattform für digitale Werkverzeichnisse via arthistoricum.net ${ }^{44}$ sowie mit Propylaeum-VITAE, ${ }^{45}$ einem biografischen Informationssystem zu Persönlichkeiten, die durch ihre Leistungen in der Archäologie und in den Altertumswissenschaften hervorgetreten sind. Im Unterschied zu gedruckten biografischen Lexika ist es dynamisch gestaltet und wird online kontinuierlich fortgeschrieben.

Zum Einsatz kommt die mit Förderung der DFG entwickelte und auf dem Content Management System DRUPAL aufsetzende Software WissKI, ${ }^{46}$ deren semantisches Ontologiemodell die Nutzung von CIDOC-CRM (ISO-Norm 21127), ${ }^{47}$ aber auch projektspezifische Anwendungsontologien vorsieht. Kontrollierte Vokabulare und Normdaten (GND, Getty-Thesauri etc.) werden eingebunden. Diese webbasierte und kollaborative Arbeits- und Publikationsweise und die multiplen Verbindungen von Bild und Text schaffen gegenüber bisherigen Printpublikationen neue Möglichkeiten der Visualisierung und Verbreitung stets aktueller Forschungsergebnisse. So können die zwischen den in der Datenbank erfassten Artefakten oder Personen bestehenden komplexen, geografischen, überlieferungskontextuellen, sprachlichen, inhaltlichen, ikonografischen oder editorischen Bezüge komfortabel recherchiert, visualisiert und dynamisch ausgebaut werden. Um darüber hinaus - im Sinne einer virtuellen Forschungsumgebung zukünftig wissenschaftliche Diskurse auch direkt an den Bild- und Textdaten führen zu können, wird auch hier das Annotationsmodul zum Einsatz kommen.

\section{Texte sind nicht alles: Zentrales Repositorium für digitale Medien}

Zwei- und dreidimensionale Bilder sowie Ton- und Filmdokumente sind heute zentrale Arbeitsmittel für Forschung und Lehre im Bereich der Kultur- und Geisteswissenschaften und gängige Werkzeuge der Digital Humanities. Die

$44 \mathrm{https}$ ://www.arthistoricum.net/themen/wvz.

45 https://www.propylaeum.de/themen/propylaeum-vitae. $46 \mathrm{http}: / /$ wiss-ki.eu.

$47 \mathrm{http}: / /$ www.cidoc-crm.org.
Objekt- und Multimediadatenbank heidICON, 2005 als „Virtuelle Diathek“ der Heidelberger Universität eingerichtet, ist heute ein unverzichtbares Repositorium und neben einer wichtigen Präsentationsplattform für digitale Medien auch Rückgrat für andere Dienste (s.o.) und damit Teil der modernen Forschungsinfrastruktur für die heterogene Wissenschaftslandschaft der Universität Heidelberg. Insbesondere für die Geisteswissenschaften und universitären Sammlungen hat die objektorientierte Datenbank, die hinsichtlich Technik und Erschließung auf internationale Standards setzt, unmittelbaren Nutzen. ${ }^{48}$

Durch ein ausgefeiltes und eng am CIDOC-CRM-Standard orientiertes Datenmodell wird heidICON den Anforderungen unterschiedlicher Forschungsdisziplinen gerecht. Es ermöglicht, die Tiefenerschließung innerhalb einzelner Datensätze und die Datensätze untereinander (hierarchisch und horizontal) in Beziehung zu setzen. Das Mapping der Erschließungsdaten auf das XML-Schema LIDO $^{49}$ ermöglicht heidICON ein hohes Maß an nationaler und internationaler Vernetzung und Sichtbarkeit. Die Einbindung von kontrolliertem Vokabular und auch die Möglichkeit der Vergabe von DOIs (Digital Object Identifiers) sowie die Bereitstellung der Inhalte via IIIF fördert die Referenzierbarkeit und die Bereitstellung der Inhalte als Linked Data im Semantic Web. ${ }^{50}$

Neben dem Download der Multimediadateien ist der Export der Metadaten in CSV, XML oder JSON-Formaten möglich oder die Erstellung von Präsentationen und deren Download. Sämtliche Funktionen in heidICON lassen sich über eine Programmierschnittstelle (API) steuern, was eine bessere Vernetzung mit bestehenden Systemen für die Datenpräsentation und die Archivierung zur Folge hat.

\section{Open Science: Infrastrukturen für die Publikation und Archivierung von Forschungsdaten}

Ergänzt werden die beschriebenen spezifischen Plattformen durch heiDATA, ein generisches Forschungsdaten-

48 Im Einsatz ist die Software easydb der Programmfabrik, https:// www.programmfabrik.de/easydb. Kürzlich erfolgte der Umstieg auf die neue Version 5.

49 Lightweight Information Describing Objects http://network.icom. museum/cidoc/working-groups/lido/what-is-lido.

50 heidICON fungiert darüber hinaus auch als Aggregator für die Bereitstellung in nationalen und internationalen Datenbanken (u. a. Prometheus, Graphikportal, Deutsche Digitale Bibliothek, Europeana). 
repositorium, das seit 2014 unter dem Dach des Kompetenzzentrums Forschungsdaten (gemeinschaftlich betrieben von der Universitätsbibliothek und dem Universitätsrechenzentrum) auf Basis der Software Dataverse ${ }^{51}$ betrieben wird. Es ist für die Unterbringung von Datensammlungen aus allen Fächern bestimmt und wird - wie die Erfahrungen der ersten Jahre gezeigt haben - auch über alle Fakultäten hinweg genutzt.

Einen wichtigen Baustein für die gesamte Infrastruktur bildet die Langzeitarchivierung der publizierten Daten. Das derzeit noch im Aufbau befindliche OAIS-konforme Langzeitarchiv-System heiARCHIVE, das gemeinsam vom Universitätsrechenzentrum und der Universitätsbibliothek Heidelberg entwickelt und zukünftig auch betrieben wird, soll seinen Betrieb im zweiten Quartal 2019 aufnehmen. Es baut auf der Erfahrung mit der bisherigen Archivierung der Retrodigitalisate der Universitätsbibliothek auf. Wesentliche Komponenten bilden dabei die Open-Source-Datenmanagement-Software iRODS ${ }^{52}$ sowie ein selbst entwickelter Ingest-Workflow.

\section{Die Heidelberger modulare Forschungsinfrastruktur}

Aus der hier skizzierten Entwicklung ist erkennbar, dass die von der UB Heidelberg für die (nicht nur digitalen) Geisteswissenschaften bereit gestellte Infrastruktur (heiRIS - Heidelberg Research Infrastructure) nicht eine homogene, in sich geschlossene Universalplattform darstellt. Es handelt sich vielmehr um ein strategisch über bald zwei Jahrzehnte hinweg aufgebautes und dadurch auch historisch gewachsenes „Ökosystem“ miteinander verzahnter Dienste, wie sie oben vorgestellt wurden (Abb. 4). Die in diesen verteilten Systemen erfassten und archivierten Inhalte werden im Südwestdeutschen Bibliotheksverbund (SWB) bzw. im Verbund K10plus bibliografisch erfasst und sind so übergreifend national und auch international auffindbar und rezipierbar.

Die weitere strategische Ausrichtung der Bibliothek auf Verlagstätigkeiten hat auch organisatorische Konsequenzen. So wurde ebenfalls 2015 eine neue Abteilung gegründet, die unter der Bezeichnung „Publikationsdienste“ die vielfältigen Aktivitäten im Haus bündelt, systematisch verlegerische Prozessabläufe definiert, Geschäftsmodelle entwickelt und in enger Zusammenarbeit mit der

51 https://dataverse.org.

52 https://irods.org.
Abteilung für Informationstechnologie zukunftsweisende technische Verfahren und Workflows erarbeitet. Durch die Einstellung von Mitarbeitern mit verlagsspezifischen Qualifikationen wurden die Kompetenzen in diesem Bereich wesentlich erhöht.

Die UB Heidelberg situiert sich damit als zentrale Einrichtung einer forschungsstarken Universität in einer nationalen und internationalen Landschaft wissenschaftlicher Infrastrukturen, die - wie auch die Wissenschaft selbst - von einer zunehmenden Vernetzung geprägt ist. Um als zuverlässige Akteurin in diesem Netzwerk zu fungieren und ihrer Rolle darin gerecht zu werden, setzt sie auf den Aufbau und Betrieb robuster lokaler Dienste, die zwar institutionell und geografisch verankert, jedoch keineswegs allein auf den Standort Heidelberg eingeschränkt sind.

Nutznießer dieser nachhaltigen Speicherorte, Forschungsinfrastrukturen und Präsentationsplattformen für die Tiefenerschließung, Archivierung und wissenschaftliche Nutzung sind nicht nur die universitären Einrichtungen und Projekte der Universität Heidelberg. Im Kontext ihres nationalen Auftrags im Rahmen der DFG-geförderten „Fachinformationsdienste für die Wissenschaft“ (FID) versorgt die UB Heidelberg auch die Fachcommunity der Heidelberger FIDs arthistoricum.net (Kunstgeschichte), Propylaeum (Altertumswissenschaften) und CrossAsia (Asienwissenschaften) mit diesen Angeboten zur Open-Access-Veröffentlichung. War es anfangs nicht einfach, Wissenschaftler von deren Vorteilen zu überzeugen, stellen die FIDs heute eine vielgenutzte Publikationsinfrastruktur für Projekte und Publikationsvorhaben der jeweiligen Fächer dar. Diverse strategische Partnerschaften mit Kultureinrichtungen und Verlagen belegen, dass die Angebote den Bedürfnissen der Fachcommunity entsprechen.

Weiteres Entwicklungspotential liegt zudem in der Mitarbeit bei den sich im Kontext der Nationalen Forschungsdateninfrastruktur (NFDI) ${ }^{53}$ aktuell formierenden Konsortien. Ziel ist der gemeinsame Aufbau einer dezentralen, fach- und forschungsnahen digitalen Forschungsinfrastruktur mit Ressourcen, Diensten und Werkzeugen für die Erforschung von Kulturgütern.

Für den Auf- und Ausbau dieser Infrastruktur, welche einen integralen Bestandteil des Zukunftskonzeptes der UB Heidelberg darstellt, ist eine ausgeprägt induktive Bottom-up-Strategie charakteristisch, bei der durch die Bedarfe sorgfältig ausgewählter Pilotprojekte die benötigten Systemkomponenten und -funktionalitäten benannt und aufgebaut werden. Dadurch werden Dienstentwicklungen

53 http://www.rfii.de/de/themen. 


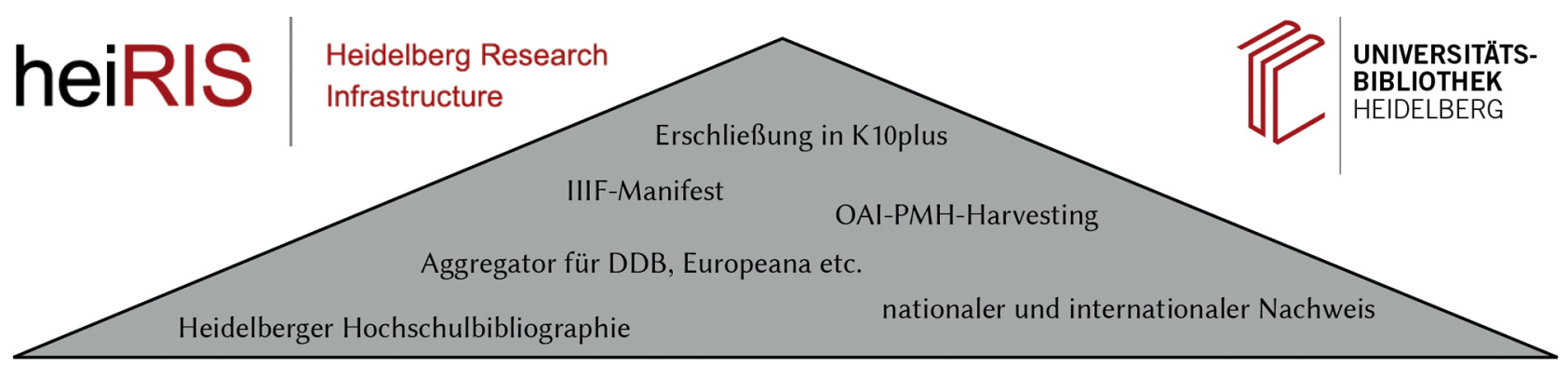

OPEN ACCESS
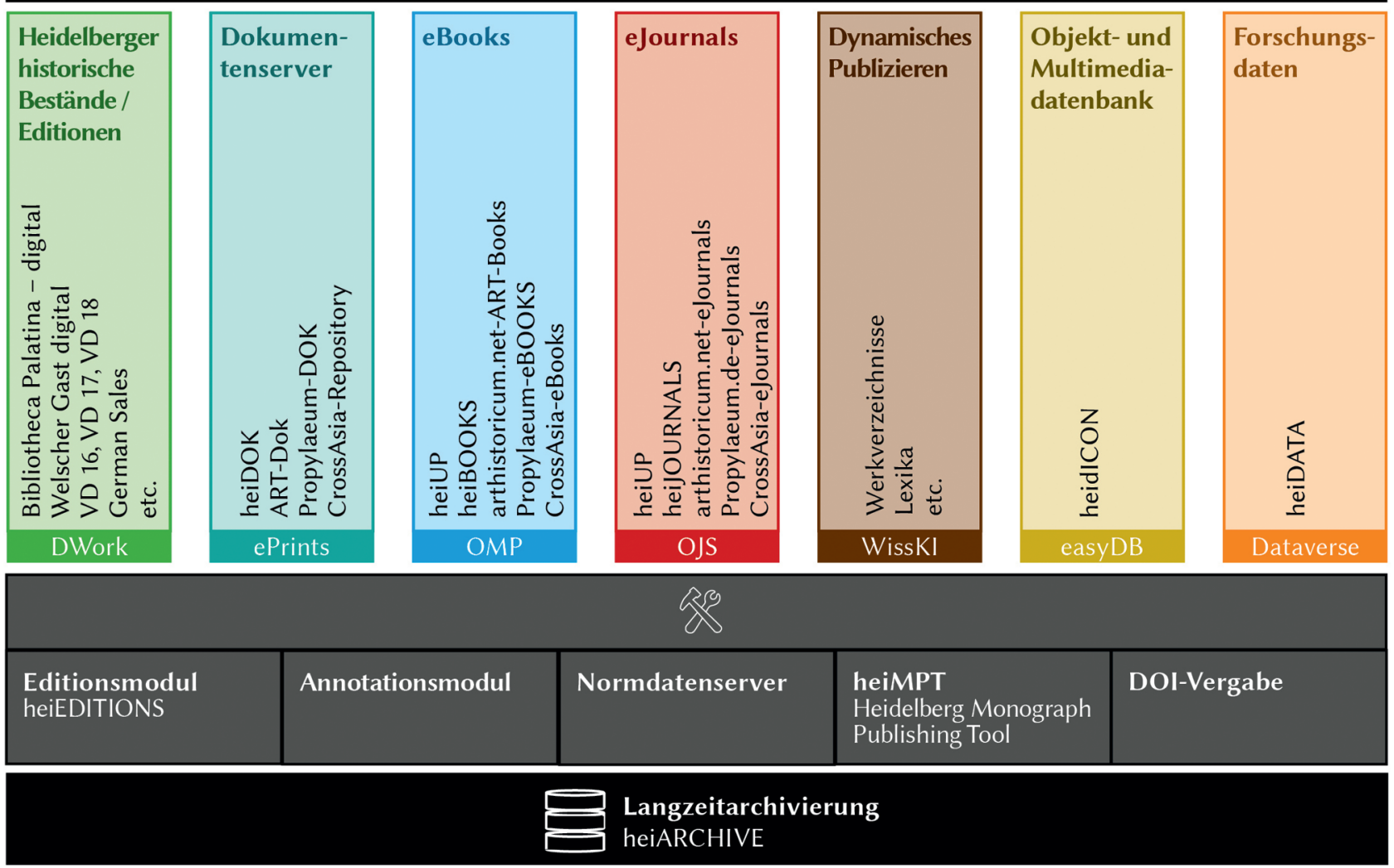

heiRIS - Die modulare Forschungsinfrastruktur der Universitätsbibliothek Heidelberg

Abb. 4: Die modulare Forschungsinfrastruktur der Universitätsbibliothek Heidelberg

„an der Wissenschaft vorbei“ vermieden. Das Risiko solcher Fehlentwicklungen wird insbesondere in allzu generischen Werkzeugen gesehen, die in gut gemeinter Absicht auf breite Nachnutzung spezifische Fachgemeinschaften am Ende nicht erreichen. Gleichzeitig ist aber klar, dass vor allem in Bezug auf die Akzeptanz und mögliche Nachnutzung der Forschungsergebnisse in der jeweiligen Fachcommunity - die Anbindung an Standardformate und Normdaten ein unabdingbares Gebot ist. Zwischen diesen Desideraten gilt es, ein Gleichgewicht zu halten.

\section{Literaturverzeichnis}

Effinger, Maria (2010): Palatina goes online. Handschriftendigitalisierung an der Universitätsbibliothek Heidelberg. In: Schnetzer, Norbert (Hrsg.): Trends, Megatrends, Sackgassen. Die Sondersammlungen im 21. Jahrhundert. Graz-Feldkirch: Neugebauer, 78-84.

Effinger, Maria (2011): Das Verborgene sichtbar machen. Neue Vermittlungs- und Vernetzungsmöglichkeiten durch die Digitalisierung mittelalterlicher Handschriften. In: Gardt, Andreas; Schnyder, Mireille; Wolf, Jürgen (Hrsg.): Buchkultur und Wissensvermittlung in Mittelalter und Früher Neuzeit. Berlin [u. a.]: De Gruyter, 55-67.

Effinger, Maria (2013): Open Access - Open Antiquity. Elektronisches Publizieren an der Universitätsbibliothek Heidelberg im Kontext 
der Virtuellen Fachbibliothek Altertumswissenschaften Propylaeum. In: Mitteilungen des Deutschen ArchäologenVerbandes, 44 (2), 52-56.

Effinger, Maria (2018): Wissen verbreiten - im Open Access publizieren: Infrastrukturen für die digitale Kunstgeschichte. In: Kuroczyński, Piotr; Bell, Peter; Dieckmann, Lisa (Hrsg.): Computing Art Reader. Einführung in die digitale Kunstgeschichte. Heidelberg: arthistoricum.net, 269-85. doi:10.11588/arthistoricum.413.596.

Effinger, Maria; Bonte, Achim (2001): HeiDok - der Publikationsserver der UB für Dissertationen und sonstige wissenschaftliche Schriften. In: Theke aktuell, 8 (3), 120-22.

Effinger, Maria; Büttner, Alexandra (2015): Open Access - Open Archaeology. Wissenschaft und Bibliothek als Dream-Team? In: Archäologische Informationen, 38, 73-82.

Effinger, Maria; Büttner, Alexandra; Kautz, Michael; Krenn, Margit (Mitwirk.) (2012): Das Verborgene sichtbar machen. Die virtuelle Rekonstruktion der Klosterbibliothek Lorsch. Ubstadt-Weiher [u. a.]: Verlag Regionalkultur.

Effinger, Maria; Krabbes, Frank; Withanage, Dulip (2018): Crossmediales Publizieren bei Heidelberg University Publishing (heiUP). In: BIT online, 21 (5), 393-403.

Effinger, Maria; Krenn, Margit; Wolf, Thomas (2008): Der Vergangenheit eine Zukunft schaffen. Die Digitalisierung der Bibliotheca Palatina in der Universitätsbibliothek Heidelberg. In: BIT online, 11(2), 157-66.

Effinger, Maria; Maylein, Leonhard; Pietzsch, Eberhard; Spyra, Ulrike (2003): Per Mausklick ins Spätmittelalter: Digitalisierung und Erschließung spätmittelalterlicher Bilderhandschriften aus der Bibliotheca Palatina. In: BIT online, 6 (3), 235-47.

Effinger, Maria; Saurma, Lieselotte E.; Pietzsch, Eberhard (2000): Deutsche Forschungsgemeinschaft fördert Projekt „Digitalisierung spätmittelalterlicher Bilderhandschriften aus der Bibliotheca Palatina“. In: Theke, 47-50.

Effinger, Maria; Schmidt, Claudia (2009): Neues DFG-Projekt: Digitalisierung und Erschließung illustrierter Kunst- und Satirezeitschriften des 19. und frühen 20. Jahrhunderts. In: AKMB-news, 15 (2), 32-33.

Effinger, Maria; Wolf, Thomas (2005): HeidICON - Die zentrale Bilddatenbank der Universität Heidelberg. In: Theke, 18-25.

Effinger, Maria; Zimmermann, Karin (2015): Bibliotheca Palatina digital. La reconstitution virtuelle d'une bibliothèque célèbre. In: Francia, 42, 291-306. doi:10.11588/fr.2015.0.44578.

Maylein, Leonhard; Effinger, Maria (2004): Digitalisierung an der Universitätsbibliothek Heidelberg am Beispiel des DFG-Projekts „Digitalisierung spätmittelalterlicher Bilderhandschriften aus der Bibliotheca Palatina“. In: Praxis der Informationsverarbeitung und Kommunikation, 27 (1), 15-17.

Saurma-Jeltsch, Lieselotte E.; Effinger, Maria (2001): Forschung per Mausklick. In: Ruperto Carola, 3, 4-12.

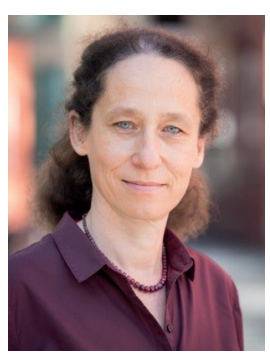

\section{Dr. Maria Effinger}

Universitätsbibliothek

Plöck 107-109

D-69117 Heidelberg

Effinger@ub.uni-heidelberg.de

orcid.org/0000-0001-6396-4876

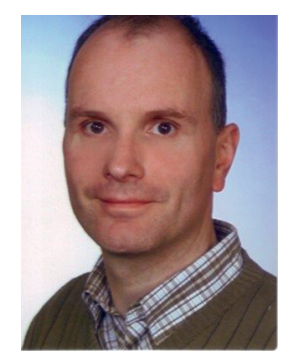

Leonhard Maylein

Universitätsbibliothek

Plöck 107-109

D-69117 Heidelberg

maylein@ub.uni-heidelberg.de

orcid.org/0000-0002-9423-489X

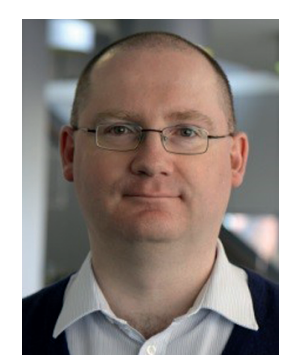

Dr. Jakub Šimek

Universitätsbibliothek

Plöck 107-109

D-69117 Heidelberg

simek@ub.uni-heidelberg.de

orcid.org/0000-0002-7262-7342 